\title{
Predictive factors of thrombosis for patients with essential thrombocythaemia: A single center study*
}

\author{
Iwona Prajs ${ }^{A-\mathrm{F}}$, Kazimierz Kuliczkowski ${ }^{\mathrm{F}}$ \\ Department and Clinic of Hematology, Blood Neoplasms, and Bone Marrow Transplantation, Wroclaw Medical University, Poland \\ A - research concept and design; B - collection and/or assembly of data; C - data analysis and interpretation; \\ $\mathrm{D}$ - writing the article; $\mathrm{E}$ - critical revision of the article; $\mathrm{F}$ - final approval of article
}

\section{Address for correspondence \\ Iwona Prajs \\ E-mail: iwona.praj@@umed.wroc.pl}

\section{Funding sources}

This study was supported by the grant of the

National Center of Sciences (NN 519314 035)

to prof. Kazimierz Kuliczkowski.

Conflict of interest

none declared

Received on January 04, 2017

Revised on January 10, 2017

Accepted on January 19, 2017

\begin{abstract}
Background. Thrombotembolic complications are the leading cause of mortality in essential thrombocythemia (ET), but the definition of thrombotic risk remains far from clear.

Objectives. The aim of this study was to evaluate the prognostic markers for thrombosis to identify ET patients at risk.

Material and methods. Forty-five consecutive patients with ET were studied. This group was divided into two subgroups ET patients with (A) and without (B) history of thrombosis. Each patient has been tested for complete blood count, fibrinogen, factor VIII, D-dimer, protein C, APCR, TAT and F1+2. JAK2 mutation was assessed by RT-PCR. Factor V Leiden and prothrombin genes mutations were screened by DNA sequencing.

Results. The median age of ET patients was 62.0 years. JAK2 mutation was found in 24 patients, 21 of them had a history of thrombotic events, and 17/21 were JAK2 positive. Compared to controls, ET patients had a significantly higher WBC and PLT counts, and higher mean platelet volume (MPV), but not Hgb level or RBC count. In ET subgroup A, apart from changes seen in the whole ET, the Hgb level, RBC count, and Hct were also significantly elevated. Interestingly, the MPV was significantly larger in subgroup A, but not in B. Fibrinogen and D-dimers levels were significantly higher in ET group than in controls, but not F1+F2 or TAT. The results of hemostatic tests did not markedly differ between subgroups A and B. APCR was found in $5 / 45$ patients with $\mathrm{ET}$, and 2 out of 5 had a factor $V$ Leiden heterozygous mutation. No prothrombin gene mutation was observed.
\end{abstract}

Conclusions. Our results suggest that MPV can serve as a simple test for assessing the hypercoagulable state in ET patients. It has been confirmed that JAK2 mutation and leukocytosis are independent predictors for thrombotic events in ET patients.

Key words: essentials thrombocythaemia, thrombosis, JAK2 mutation, platelets, leukocytes

DOI

10.17219/acem/68578

Copyright

Copyright by Author(s)

This is an article distributed under the terms of the

Creative Commons Attribution Non-Commercial License

(http://creativecommons.org/licenses/by-nc-nd/4.0/) 


\section{Introduction}

Essential thrombocythemia (ET) is a stem cell disorder whose distinguishing characteristic is a markedly elevated platelet count caused by excessive megakaryocyte proliferation. The activating JAK2V617F mutation is seen in nearly $60 \%$ of ET patients ${ }^{1}$, CALR mutation in about $20-$ $25 \%, c-M P L$ gene mutation in a few percent, and about $10 \%$ to $25 \%$ are defined as "triple negative".2,3 An annual incidence rate in the United States is approximately 0.5 to 1.5 cases per 100,000 persons per year. The median age at diagnosis is approximately in the mid-seventh decade of life, but it can occur cross in all age groups, and there is a 1.5 to 2 -fold female predominance, especially in those diagnosed in the third to fifth decade of life.

Thrombotic complications are present at diagnosis in about $9-22 \%$ of patients. Thromboembolic risk has been associated with age ( $>60$ years), prior thrombosis, leukocytosis, and possibly also with JAK2V617F mutation. ${ }^{4}$

Morbidity and mortality from ET in the first decade of the disease are predominantly related to thrombotic, vasomotor, and less commonly, to hemorrhagic complications. Long standing ET can progress to myelofibrosis or acute myeloblastic leukemia. ${ }^{5}$

\section{Material and methods}

This study included 45 consecutive patients with essential thrombocythemia (ET) attending the out-patient clinic at the Department of Hematology, Blood Neoplasms, and Bone Marrow Transplantation in Wroclaw, Poland. The diagnosis of ET was made on the basis of the 2008 World Health Organization criteria. ${ }^{6}$ Among the 45 ET patients, there were 14 men and 31 women; the age range was between 25-89 years. Neither of the ET patients had a new thrombotic complication at the time of entering the study. A group of 45 healthy subjects (18 men, 27 women; age range, $22-78$ years) was recruited from healthy adults without history of thrombotic events to serve as a control group.

All investigations were approved by the Bioethics Commission of Wroclaw Medical University, Poland. The study was carried out in accordance with the Declaration of Helsinki of 1975, as revised in 2000. Written informed consent was obtained from all patients and controls before entering the study.

The characteristics of the patients and the control subjects were summarized in Table 1. Of 45 ET patients, 12 asymptomatic patients did not receive any treatment for ET, 22 were treated with anagrelide (thromboreductin), 8 with hydroxyurea (HU), and 3 with aspirin (ASA). Twenty-one patients had thrombotic events in the past. Fifteen patients had arterial thrombosis: 6 patients had myocardial infarction (MI), 7 patients had transient ischemic attacks (TIA), 4 patients had peripheral arterial thrombosis (PAT) of the
Table 1. Characteristic of patients with essential thrombocythaemia (ET)

\begin{tabular}{|l|c|c|}
\hline Data/Group & $\begin{array}{c}\text { Essential } \\
\text { thrombocythaemia } \\
(n=45)\end{array}$ & $\begin{array}{c}\text { Controls } \\
(\mathrm{n}=45)\end{array}$ \\
\hline $\begin{array}{l}\text { Age: median } \pm \mathrm{SD} \\
\text { (range) } \\
\begin{array}{l}\text { Gender } \\
\text { (male/female) }\end{array}\end{array}$ & 52.0 (range $25-89)$ & $18 / 27$ \\
$\begin{array}{l}\text { JAK2 positive } \\
\text { (male/female) }\end{array}$ & $14 / 31$ & 0 \\
$\begin{array}{l}\text { Thromboembolic events } \\
\text { (male/female) }\end{array}$ & $24(10 / 14)$ & 0 \\
\hline
\end{tabular}

ET - essential thrombocythaemia; JAK2 mutation - Janus kinase (JAK2) V517F mutation.

legs, and 6 patients had deep venous thrombosis (DVT). Microvascular disturbances and superficial thrombophlebitis of the legs were not considered in this analysis.

\section{Methods}

Each patient has been tested by automated methods for blood morphology (Sysmex). Plasma fibrynogen level, Ddimer level, factor VIII activity, protein $\mathrm{C}$ level, activated protein $C$ resistance (APCR) were measured in coagulometer Ceverone alpha (Technoclone). Plasma concentrations of thrombin-anti-thrombin complexes (TAT) and prothrombin fragment $\mathrm{F}_{1+2}(\mathrm{~F} 1+2)$ were determined by ELISA, using commercially available kits (Enzygnost TAT and Enzygnost F1+2, respectively; Siemens).

Testing for the factor V Leiden (G11691A) gene and prothrombin gene (FII G20210A) hereditary mutations were screened by DNA sequencing.

$J A K 2$ mutation was screened by the real-time RT-PCR assay.

The applied dispersed computer system was used for the accumulation and safety storage of patients' data and the results of the laboratory tests.

\section{Statistical analysis}

All calculations were performed with the R Project for Statistical Computing, with the threshold of statistical significance set at a p-value of 0.05 or lower.

Patients' characteristics were compared with the use of the two-tailed Fisher's exact test for categorical variables, and the Mann-Whitney U test for continuous variables.

\section{Results}

The study population consisted of 45 subjects with ET, 14 males and 31 females, with the median age of 62.0. Their general characteristics are presented in Table 1. Among the 45 ET patients, 24 tested positive for the JAK2 heterozygous mutation, predominantly among fe- 
males 14/24. Thromboembolic events were reported in 21 out of 45 ET patients, in 6 males and in 15 females.

The comparison of blood picture parameters and hemostatic tests in ET and the control group are shown in Table 2 .

Leukocyte $\left(\mathrm{p}<10^{-6}\right)$ and platelet $\left(\mathrm{p}<10^{-6}\right)$ counts were significantly higher, and MPV markedly bigger ( $\mathrm{p}=0.0001)$ in ET patients than in healthy subjects, but not hemoglobin level or erythrocyte count.

Fibrinogen concentration $(\mathrm{p}=0.0015)$ and D-dimers $\left(\mathrm{p}<10^{-6}\right)$ were significantly higher in the ET group than in controls, but not F1 + F2, TAT, factor VIII activity, PC activity and activated protein $\mathrm{C}$ resistance.

APCR was found in 5/45 ET patients with ET and in 2/45 controls. None of ET patients were carriers of the FV Leiden homozygous mutation, but two ET patients had the FV Leiden heterozygous mutation and 2 controls. Prothrombin gene mutation (G20210A) was not present in either group.

Compared to the control groups, ET patients with thrombosis (A) had significantly elevated leukocyte $\left(\mathrm{p}<10^{-6}\right)$ and platelets $\left(\mathrm{p}<10^{-11}\right)$ counts, MPV $\left(\mathrm{p}<10^{-8}\right)$, D-dimers level (0.0001).

Table 4 shows that in ET patients without thrombosis (B) the hemoglobin level $(\mathrm{p}=0.003)$, leukocyte count $\left(\mathrm{p}<10^{-10}\right)$, and platelet count $\left(\mathrm{p}<10^{-10}\right)$ were significantly higher than in controls, but not MPV ( $\mathrm{p}=0.771)$. In the ET group without thrombosis (subgroup B) the fibrinogen $(\mathrm{p}=0.004)$ and D-dimers $\left(\mathrm{p}<10^{-6}\right)$ levels were also significantly elevated by comparison with the control groups.

As shown in Table 5, the blood count differs significantly between ET patients with (A) and without (B) thrombosis. The hemoglobin level ( $p=0.0002)$, erythrocyte count $(p=0.01)$, hematocrit $(p=0.012)$, leukocyte count $(p=0.0033)$ and platelet count $(p=0.046)$ were significantly increased in ET patients with thrombosis (A). The mean platelet volume was significantly larger in ET subgroup with (A) than that in the subgroup (B) without thrombosis $\left(\mathrm{p}<10^{-8}\right)$.

The coagulation tests did not shown any statistically significant differences concerning fibrynogen, D-dimers, factor VIII activity, protein C activity, APCR, F1+F2 and TAT levels between two ET subgroups with (A) and without (B) thrombosis.

\section{Discussion}

We have confirmed that the occurrence of $J A K 2$ mutation can be a strong predictor for thromboembolic events in ET patients. Seventeen out of 21 ET patients with thrombotic complications were $J A K 2(+)$. Since its discovery in 2005 , the $J A K 2$ mutation has been considered as an important participant in the development of thrombotic complications in Ph-myeloproliferative neoplasms, but the agreement was not universal. ${ }^{1}$ Carobbio et al. in 657 studied ET patients did not find a significant association for $J A K 2$ mutation and the frequency of thromboembolic events. ${ }^{7}$ De Stefano et al. confirmed that in JAK2 (+) ET patients aged $<60$ years the risk of thrombosis increased 3.8 fold in the presence of homozygous and 2.2 fold in the case of a hetrozygous type of FV Leiden mutation. ${ }^{8}$ The systematic review and meta-analysis of 2,436 patients with ET shows that $J A K 2$ positivity correlated with a clear increase in the odds of thrombosis $(\mathrm{OR}=1.83)^{9}$, and this was also observed by others. ${ }^{10-12}$ Our findings are in line with the results of Schwartz et al., ${ }^{13}$ who have proven, on the basis of an analyzed cohort of 1,179 thrombocythemic Ph-myeloptroliferations, that JAK2 mutation affected significantly the incidence of thrombosis (both arterial and venous). The JAK2 mutation has been included recently into the major criteria for $\mathrm{ET}^{14,15}$, and is also considered as a major prothrombotic risk factor in the International Prognostic Score in Essential Thrombocythemia (IPSET) risk factor study in WHO-defined ET. ${ }^{16}$

It is not easy to analyze laboratory results obtained in our patients with ET, as it is necessary to consider that some results might have been conflated not only by the disease itself, but also by the treatment effects of anagrelide, hydroxyurea or ASA. Many other investigators faced similar problems.

In ET patients, leukocyte and platelet counts were found to be significantly higher, and the mean platelet volume (MPV) markedly bigger than in healthy subjects, but not hemoglobin level or erythrocyte count.

However, in the subgroup of ET patients with thrombotic events (A), apart from significantly elevated platelet and leukocyte counts and higher MPV, the hemoglobin level, hematocrit, and erytrocyte counts were also increased significantly compared with that in ET patients without thrombosis (B). Belluci and Michiels suggest that heterozygous $J A K 2$ mutation can be responsible for the increased hemoglobin and hematocrit in some ET patients. ${ }^{17}$ Rotunno $\mathrm{G}$ et al. found that JAK2(+) ET patients have a higher $\mathrm{Hb}$ level and are more prone to thrombotic events than CALR (+) ET patients. ${ }^{3}$

Further analysis of our results revealed that between the ET subgroups with (A) and without thrombosis (B) the statistical change in the leukocyte count was much stronger $(\mathrm{p}=0.0033)$ than in the platelet count $(\mathrm{p}=0.046)$. Leukocyte count $>11 \mathrm{~g} / \mathrm{L}$ is an established risk factor for thrombosis in ET. It shall be stressed that the median platelet count in subgroup A was $676.0 \mathrm{~g} / \mathrm{L}$ (ranged from $544.0 \mathrm{~g} / \mathrm{L}$ to $837.0 \mathrm{~g} / \mathrm{L}$ ), but in subgroup B though, the median platelet count was $535.0 \mathrm{~g} / \mathrm{L}$. This subgroup was much more heterogeneous as platelet counts ranged from $300.0 \mathrm{~g} / \mathrm{L}$ to $2022.0 \mathrm{~g} / \mathrm{L}$.

Interestingly, the presence of bigger, younger and more hemostatically active platelets caused a significant increase in the mean platelet volume in all ET patients and in the subgroup of ET with thrombosis, but not in ET pa- 


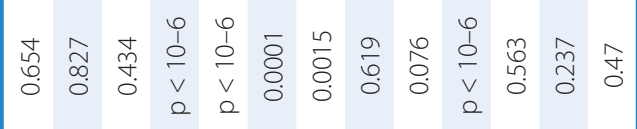

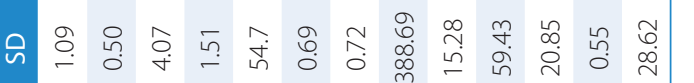

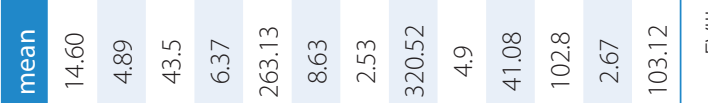

多

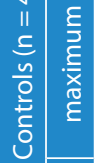

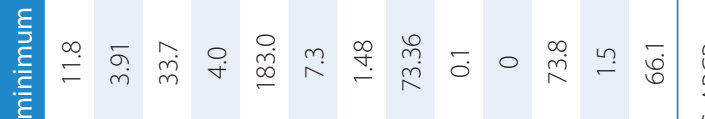

高告

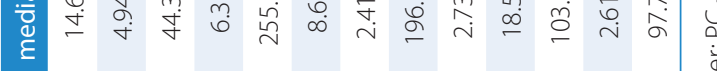

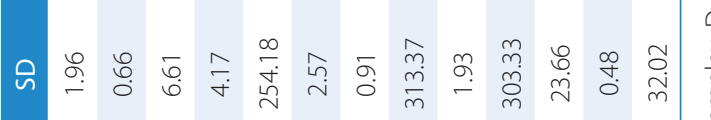

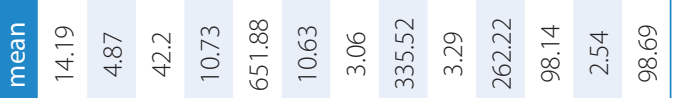

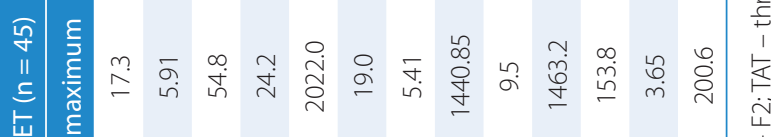

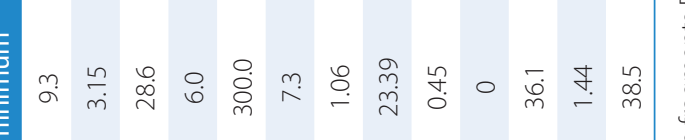

热

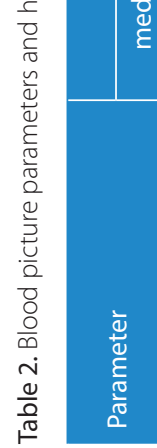

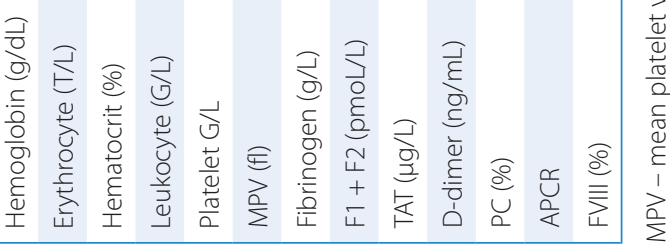

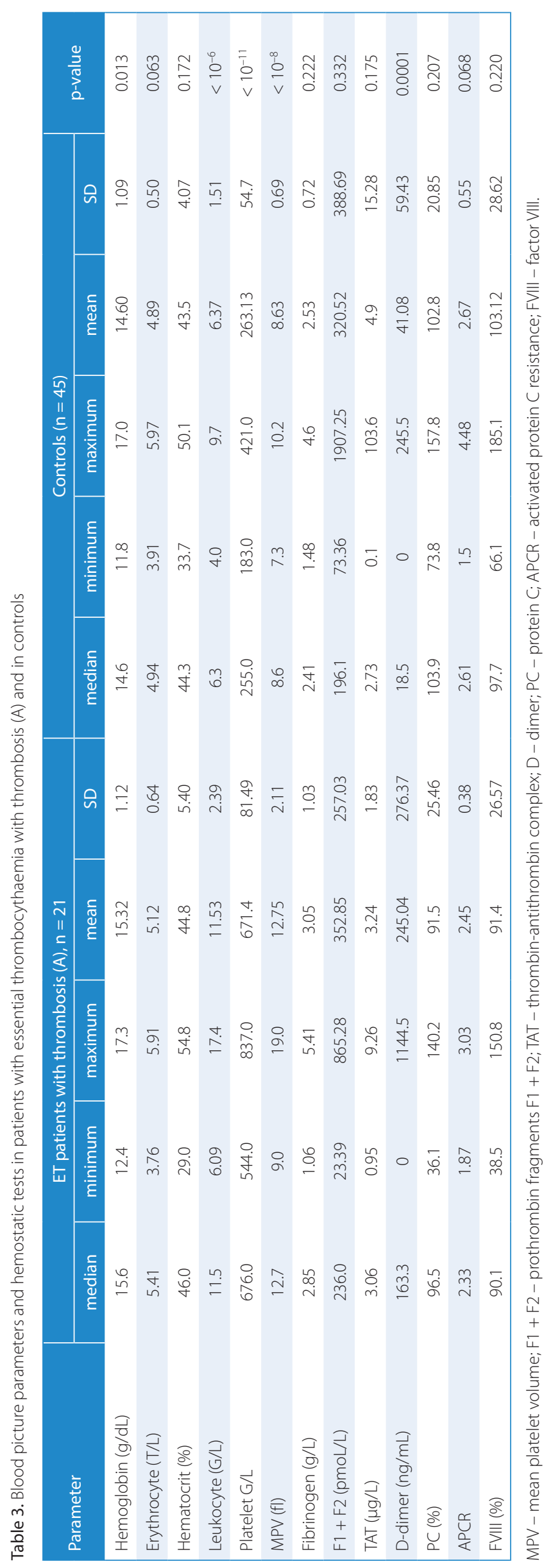




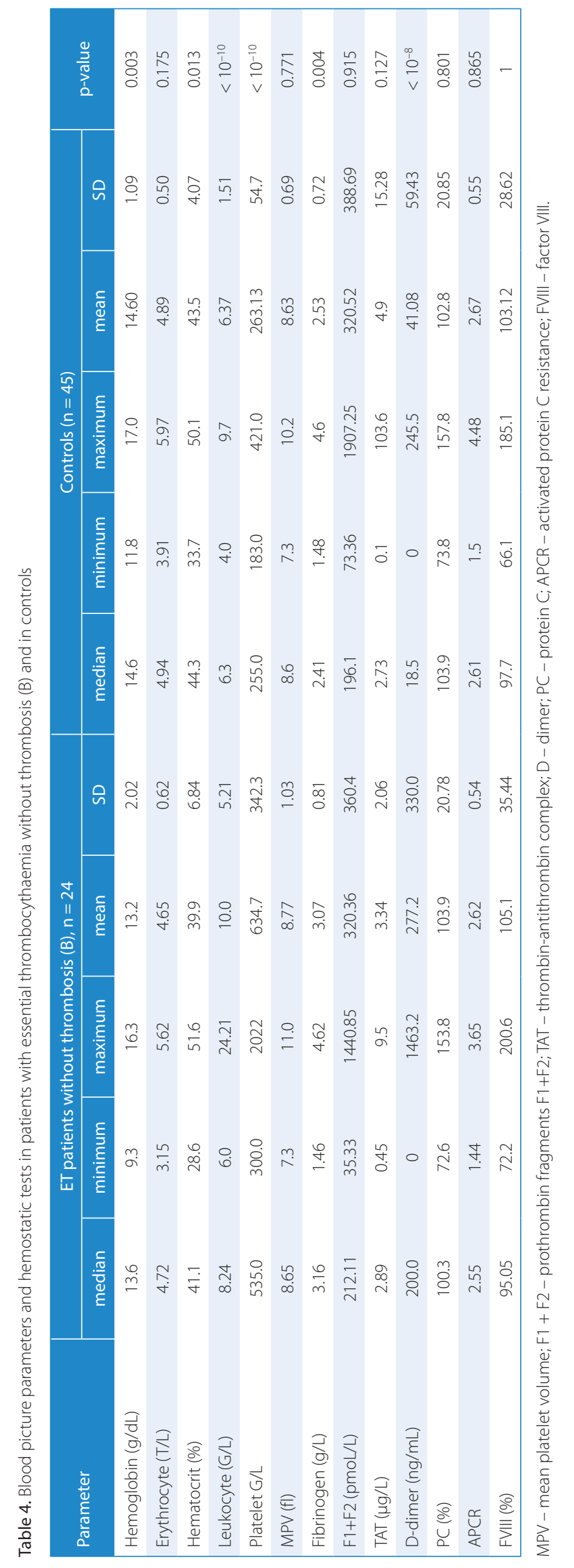

要,

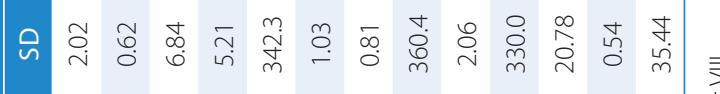

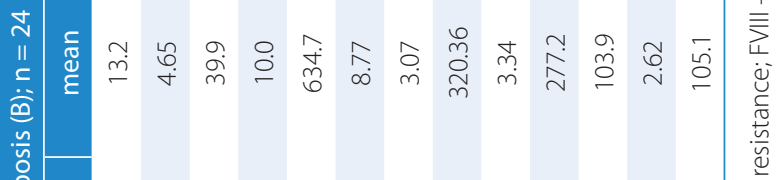

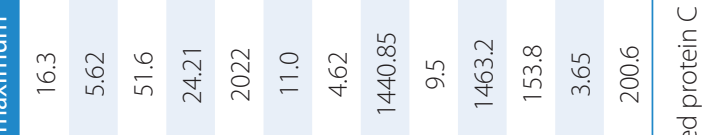

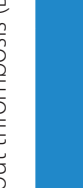

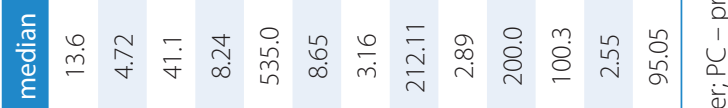

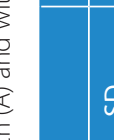

$\frac{1}{15}$

竞

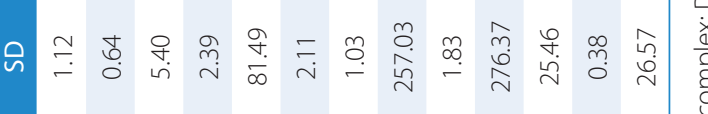

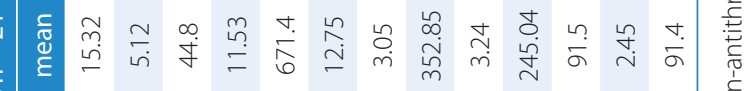

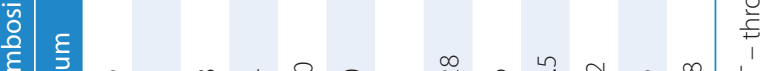

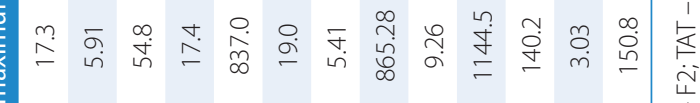

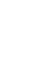


tients without thrombosis. If confirmed in a larger group of ET patients, MPV can possibly be useful in predicting a prothrombotic state. Arellano-Rodrigo et al. ${ }^{18}$ observed that ET patients had higher values of MPV, especially those with $J A K 2$ mutation. ${ }^{18}$ At a multivariate study it has been confirmed that ET patients with thrombosis had a significantly higher percentage of reticulated platelets. Increased neutrophil and platelet activation play an important role in the pathogenesis of thrombotic complications in ET patients. ${ }^{19-22}$

We have observed that both fibrinogen $(\mathrm{p}=0.0015)$ and D-dimer $\left(\mathrm{p}<10^{-6}\right)$ levels were significantly higher in our ET patients than in controls, but they did not differ between ET subgroups with and without thrombosis. Blann et al. ${ }^{23}$ observed some elevation in the fibrynogen level in treated and untreated patients with ET, but it remained within normal ranges.

Some authors described elevated D-dimer levels in patients with ET at diagnosis or treated with ASA or hydroxyurea and $\mathrm{ASA}^{23,24}$, but others have shown its reduction after cytoreductive therapy. ${ }^{25}$ However, the increased D-dimer level has not been proven as a clinically useful predictor for thrombosis, when compared with some other confirmed predictors such as age and history of thrombosis or leukocytosis. ${ }^{26,27}$ In patients with ET we did not find an increase in factor VIII activity, trombinanti-thrombin complexes and prothrombin fragments $\mathrm{F} 1+\mathrm{F} 2$ levels or protein $\mathrm{C}$ activity.

Our observations are not in keeping with the results of some other investigators who observed elevated F1 + F2 fragments ${ }^{19}$ in ET patients.

No statistically significant difference has been found in the frequency of factor $\mathrm{V}$ Leiden or prothrombin gene mutation compared to the control group. It is in agreement with the results of other authors. ${ }^{11,13}$ Out of five ET patients presenting APCR the factor V Leiden heterozygous mutation has been confirmed in 2, and the remaining three had acquired APCR. Both ET patients with FVL mutation experienced thrombotic events. The knowledge of carriership of FVLeiden can be helpful in the individual thrombosis risk assessment.

However, hereditary thrombophilia with essential thrombocythaemia increases the risk of thrombosis 2.7 fold in carriers and 5 fold in JAK2 (+) ET patients. ${ }^{10}$ Schwartz et al. have shown that in ET the specific thrombophilic markers, e.g. FVLeiden mutation and protein $\mathrm{C}$ deficiency, were connected with thrombotic venous events. ${ }^{13}$

In conclusion, our results strongly suggest that changes of mean platelet volume can serve as an additional and simple test in prothrombotic state assessment in ET patients, but this needs to be confirmed in a larger population. The presence of $J A K 2$ mutation and leukocytosis has been proven to be a strong predictor for thromboembolic events in ET patients.

\section{References}

1. Kralovics R, Passamonti F, Buser AS, et al. A gain-of-function mutation of JAK2 in myeloproliferative disorders. N Engl J Med. 2005; 352:1779-1790.

2. Vainchenker W, Delhommeau F, Constantinescu SN, Bernard OA. New mutations and pathogenesis of myeloproliferative neoplasms. Blood. 2011;118:1723-1735.

3. Rotunno G, Mannarelli C, Guglielmelli P, et al. Impact of calreticulin mutations on clinical and hematological phenotype and outcome in essential thrombocythaemia. Blood. 2014;123:1552-1555.

4. Tefferi A, Thiele J, Orazi A, et al. Proposals and rationale for revision of the World Health Organisation diagnostic criteria for polycythemia vera, essential thrombocythemia, and primary myelofibrosis: recommendations from an ad hoc international expert panel. Blood. 2007;110:1092-1097.

5. Tefferi A, Barbui T. Personalized management of essential thrombocythemia: application of recent evidence to clinical practice. Leukemia. 2013;27:1617-1620.

6. Tefferi A, Vardiman JW. Classification and diagnosis of myeloproliferative neoplasms: The 2008 World Health Organization criteria and point-of-care diagnostic algorithms. Leukemia. 2008;22:14-22.

7. Carobbio A, Finazzi G, Guerini V, et al. Leukocytosis is a risk stratification assessment in essential thrombocythemia. J Clin Oncol. 2008;26:2732-2736.

8. De Stefano V, Za T, Rossi E, et al. Influence of the JAK2 V617F mutation and inherited thrombophilia on the thrombotic risk among patients with essentials thrombocythemia. Haematologica. 2009; 94:733-737.

9. Dahabreh IJ, Zoi K, Giannouli S, Zoi C, Loukopoulos D, Voulgarelis M. Is JAK2 V617F mutation more than a diagnostic index? A meta-analysis of clinical outcomes in essential thrombocythemia. Lek Res. 2009;33: 67-73.

10. Amitrano L, Guardascione MA, Ames PRJ, et al. Thrombophilic genotypes, natural anticoagulants, and plasma homocysteine in myeloproliferative disorders: Relationship with splanchnic vein thrombosis and arterial disease. Ann J Hematol. 2003;72:75-81.

11. Afshar-Kharghan V, López JA, Gray LA, Padilla A, Borthakur G, Roberts SC, Pruthi RK, Tefferi A. Hemostatic gene polymorphisms and the prevalence of thrombotic complications in polycythemia vera and essential thrombocythemia. Blood Coagul Fibrinolysis. 2004;15:21-24.

12. Gisslinger $\mathrm{H}$, Rodeghiero F, Ruggeri $\mathrm{M}$, et al. Homocysteine levels in polycythaemia vera and essential thrombocythaemia. Br J Haematol. 1999;105:551-555.

13. Schwartz J, Ovesna P, Cerna O, et al. Thrombosis in thrombocythemic Ph-myeloproliferations is associated with higher platelet count prior to the event: results of analysis of prothrombotic risk factors from a registry of patients treated with anagrelide. Eur J Haematol. 2016;96:98-106.

14. Vannucchi AM, Barbui T, Cervantes F, et al. On behalf of the ESMO Guidelines Committee: Philadelphia chromosome-negative chronic myeloproliferative neoplasms: ESMO Clinical Practice Guidelines for diagnosis, treatment and follow-up. Ann Oncol. 2015; 26 Suppl 5:85-95.

15. Arber DA, Orazi A, Hasserjian R, et al. The updated WHO classification of hematological malignancies. The 2016 revision to the World Health Organization classification of myeloid neoplasms and acute leukemia. Blood. 2016;127:2391-2405.

16. Barbui T, Finazzi G, Corobbio A, et al. Development and validation of an International Prognostic Score of thrombosis in World Health Organization - Essential Thrombocythemia (IPSET-thrombosis). Blood. 2012;120:5128-5133.

17. Belluci S, Michiels JJ. The role of JAK2 V617F mutation, spontaneous erythropoiesis and megakaryocytopoiesis, hypersensitive platelets, activated leukocytes, and endothelial cells in etiology of thrombotic manifestations in polycythemia vera and essential thrombocythaemia. Semin Thromb Hemost. 2006;32:381-398.

18. Arellano-Rodrigo E, Alvarez-Larran A, Reverter JC, et al. Automated assessment of the neutrophil and platelet activation status in patients with essential thrombocythaemia. Platelets. 2012;23:336-343. 
19. Arellano-Rodrigo E, Alvarez-Larran A, Reverter JC, et al. Platelet turnover, coagulation factors, and soluble markers of platelet and endothelial activation in essential thrombocythaemia: relationship with thrombosis occurence and JAK2 V617F allele burden. Am J Hematol. 2009;84:102-108.

20. Arellano-Rodrigo E, Alvarez-Larrán A, Reverter JC, et al. Increased platelet and leukocyte activation as contributing mechanisms for thrombosis in essential thrombocythemia and correlation with the JAK2 mutational status. Haematologica. 2006;91:169-175.

21. Marchetti M, Castoldi E, Spronk HM, et al. Thrombin generation and activated protein $C$ resistance in patients with essential thrombocythemia and polycythemia vera. Blood. 2008;112:4061-4068.

22. Cervantes F, Arellano-Rodrigo E, Alvarez-Larrán. Blood cell activation in myeloproliferative neoplasms. Haematologica. 2009;94: 1484-1488.

23. Blann A, Caine G, Bareford D. Abnormal vascular, platelet and coagulation markers in primary thrombocythaemia are not reversed by treatments that reduce the platelet count. Platelets. 2004;15:447-449.
24. Falanga A, Marchetti M, Vignoli A, Balducci D, Barbui T. Leukocyteplatelet interaction in patients with essential thrombocythaemia and polycythemia vera. Exp Hematol. 2005;33:523-530.

25. Robertson B, Urquhart C, Ford I, Townend J, Watson HG, Vickers $\mathrm{MA}$, et al. Platelet and coagulation activation markers in myeloproliferative diseases: relationships with JAK2 V617 F status, clonality, and antiphospholipid antibodies. J Thromb Haemost. 2007;5:16791685.

26. Treliński J, Wierzbowska A, Krawczyńska A, Sakowicz A, Pietrucha T, Smolewski P, Robak T, Chojnowski K. Circulating endothelial cells in essential thrombocythemia and polycythemia vera: correlation with JAK2-V617F mutational status, angiogenic factors and coagulation activation markers. Int J Hematol. 2010;91792-91792-8. doi: 10.1007/s12185-010-0596-7. Epub 2010 May 15.

27. Kaifie $A$, Kirschner $M$, Wolf $D$, et al. Bleeding, thrombosis, and anticoagulation in myeloproliferative neoplasms (MPN): Analysis from the German SAL-MPN-registry. J Hematol Oncol. 2016;9:18. doi: $10.1186 / \mathrm{s} 13045-016-0242-9$. 